\section{RELAÇÃO ENTRE SOMATÓRIO DE DOBRAS CUTÂNEAS E PRESSÃO ARTERIAL SISTÊMICA EM ADOLESCENTES}

\author{
Relation between sum of skinfolds and systemic blood pressure \\ in adolescents
}

\author{
Relación del total de pliegues cutáneos y presión arterial \\ sistémica de adolescentes
}

\section{RESUMO}

Objetivo: Relacionar o somatório de dobras cutâneas com a pressão arterial sistêmica em adolescentes da rede pública. Métodos: Estudo transversal realizado com 543 adolescentes da rede pública de ensino de Curitiba-PR-Brasil, com idade entre 11 e 17 anos e independente do sexo, no período de agosto de 2010 a junho de 2011. Mediu-se peso corporal, estatura e dobras cutâneas (triciptal, subescapular, suprailiaca, abdominal e panturrilha). Mensurou-se a pressão arterial sistólica (PAS) e diastólica (PAD) através do método auscultatório, em duplicata. Realizou-se uma nova avaliação no dia posterior à primeira coleta nos adolescentes identificados com pré-hipertensão ou hipertensão. Utilizou-se a correlação parcial como medida de associação entre as variáveis, considerando estatura e idade como variáveis de controle. As análises foram estratificadas por sexo e o nível de significância foi de $5 \%$. Resultados: Entre adolescentes do sexo masculino, o somatório de dobras cutâneas teve uma correlação com a PAS e a PAD de $0,18(p<0,01)$ e $0,14(p<0,05)$, respectivamente. Entre adolescentes do sexo feminino, a correlação do somatório de dobras cutâneas com a PAS e PAD foi de $0,15(\mathrm{p}<0,01)$ e $0,19(\mathrm{p}<0,01)$, respectivamente. Da amostra total, $9,2 \%(\mathrm{n}=50)$ foram considerados pré-hipertensos e 7,6\% $(n=41)$ hipertensos. Conclusão: O somatório de dobras cutâneas apresentou relação direta com pressão arterial sistêmica nos adolescentes investigados.

Descritores: Adolescente; Dobras Cutâneas; Distribuição da Gordura Corporal; Pressão Arterial.

\section{ABSTRACT}

Objective: To relate the sum of skinfolds to systemic blood pressure in adolescents attending public schools. Methods: Cross-sectional study conducted with 543 adolescents from public schools of Curitiba-PR-Brazil, aged between 11 and 17 years, regardless of sex, in the period from August 2010 to June 2011. Body weight, height and skinfolds (triceps, subscapular, suprailiac, abdominal and calf) were measured. Systolic (SBP) and diastolic (DBP) blood pressure were determined by duplicate auscultatory method. A new evaluation was performed the day after the first collection in adolescents identified with pre-hypertension or hypertension. Partial correlation was used as a measure of association between variables, considering height and age as control variables. Analyses were stratified by sex and the level of significance was set at 5\%. Results: Among male adolescents, the sum of skinfolds presented a correlation to SBP and DBP of $0.18(p<0.01)$ and $0.14(p<0.05)$, respectively. Among female adolescents, the correlation of the sum of skinfolds to SBP and DBP was $0.15(p<0.01)$ and $0.19(p<0.01)$, respectively. Of the total sample, 9.2\% $(n=50)$ were prehypertensive and $7.6 \%(n=41)$ were hypertensive. Conclusion: the sum of skinfolds were directly related to the systemic blood pressure of the adolescents assessed.

Descriptors: Adolescent; Skinfold Thickness; Body Fat Distribution; Blood Pressure.
Artigo Original
1) Universidade Federal do Paraná - UFPR Curitiba (PR) - Brasil
Recebido em: 21/05/2013 Revisado em: 11/09/2013 Aceito em: 24/01/2014 


\section{RESUMEN}

Objetivo: Relacionar el total de pliegues cutáneos y la presión arterial sistémica de adolescentes de la red pública. Métodos: Estudio transversal realizado con 543 adolescentes de la red pública de enseñanza de Curitiba-PR-Brasil, con edad entre los 11 y 17 años y independiente del sexo en el periodo entre agosto de 2010 y junio de 2011. Se medio el peso corporal, la estatura y los pliegues cutáneos (triciptal, subescapular, suprailíaco, abdominal y pantorrilla). Se mensuro dos veces la presión arterial sistólica (PAS) y diastólica (PAD) a través del método auscultatorio. Se realizó una nueva evaluación el día después de la primera en los adolescentes identificados con pre-hipertensión o hipertensión elevada. Se utilizó la correlación parcial como medida de asociación de variables, considerando estatura y edad como variables control. Los análisis fueron estratificados por sexo y el nivel de significación fue del 5\%. Resultados: El total de pliegues cutáneos entre los adolescentes del sexo masculino se correlacionó con la PAS y la PAD en 0,18 ( $p<0,01)$ y 0,14 $(p<0,05)$, respectivamente. La correlación del total de pliegues cutáneos con la PAS y PAD de los adolescentes del sexo femenino fue de 0,15 $(p<0,01)$ e 0,19 $(p<0,01)$, respectivamente. De toda la muestra, el 9,2\% (n=50) fueron considerados pre-hipertensos y el 7,6\% (n=41) hipertensos. Conclusión: El total de pliegues cutáneos presentó relación directa con la presión arterial sistémica de los adolescentes investigados.

Descriptores: Adolescentes; Grosor de Pliegues Cutáneos; Distribución de la Grasa Corporal; Presión arterial.

\section{INTRODUÇÃO}

A hipertensão arterial sistêmica é um fator de risco importante e independente para doença cardiovascular, acidente vascular cerebral (AVC) e doença renal, demonstrando relação direta com estas condições, aumentando significativamente a morbidade e a mortalidade ${ }^{(1,2)}$. Afeta mais de 1 bilhão de pessoas em todo o mundo ${ }^{(3)}$ e, no Brasil, inquéritos de base populacional demonstram uma alta prevalência em adultos, com proporções variando de $22,3 \%$ até $43,9 \%{ }^{(2)}$.

Estas altas prevalências encontradas em adultos são preocupantes, contudo, a literatura nacional tem observado prevalências de hipertensão arterial sistêmica em crianças e adolescentes variando de $3,6 \%$ a $21,2 \%{ }^{(4-7)}$. Esta doença está associada a diversos fatores de risco ambientais como os baixos níveis de atividade física, o tabagismo, o consumo excessivo de sal e de álcool, além do histórico familiar, a diabetes e o nível socioeconômico, tanto em adultos quanto em adolescentes ${ }^{(2)}$.

Apesar desta gama de fatores de risco, o excesso de gordura corporal e seu impacto na saúde do indivíduo têm sido amplamente estudados, pois essa condição vem aumentando consideravelmente nas últimas décadas ${ }^{(8,9)}$. Esta avaliação da gordura corporal na população jovem pode ser realizada através de diversos métodos, contudo, métodos mais simples e de menor custo, como as medidas antropométricas, têm sido frequentemente empregados para estudos em grande escala ${ }^{(10)}$.

Dentre estes métodos antropométricos, o índice de massa corporal (IMC) é o indicador mais utilizado na rotina clínica e em estudos epidemiológicos ${ }^{(10)}$, no entanto, ele não estima diretamente a gordura corporal ${ }^{(11)}$. Neste sentido, a utilização do somatório de dobras cutâneas pode medir mais precisamente a massa gorda ${ }^{(10)}$, responsável pelas disfunções orgânicas como a hipertensão arterial sistêmica ${ }^{(2)}$.

A partir destas considerações, o presente estudo teve como objetivo relacionar o somatório de dobras cutâneas com a pressão arterial sistêmica em adolescentes da rede pública.

\section{MÉTODOS}

Estudo observacional com delineamento transversal foi realizado com adolescentes matriculados em turmas de $6^{\mathrm{a}}$ série do ensino fundamental ao $2^{\circ}$ ano do ensino médio de escolas da rede pública de ensino da cidade de Curitiba-PRBrasil, desenvolvido entre agosto de 2010 a junho de 2011.

A população alvo do estudo contou com 115.524 adolescentes, matriculados em turmas de $6^{\mathrm{a}}, 7^{\mathrm{a}}$ e $8^{\mathrm{a}}$ séries do ensino fundamental ( 160 escolas) e $1^{\circ}$ e $2^{\circ}$ anos do ensino médio (106 escolas), da rede pública de ensino da cidade de Curitiba-PR.

Participaram do estudo 543 adolescentes, independente do sexo e com idades entre 11 e 17 anos, de 44 escolas escolhidas aleatoriamente por sorteio. Consideraramse como critérios de exclusão: a não entrega do termo de consentimento livre e esclarecido assinado pelos pais ou responsáveis até o dia da coleta, falta de dados antropométricos, falta de reavaliação da pressão arterial, uso contínuo de contraceptivos orais, gestação, uso de medicamentos anti-hipertensivos e desistência na participação do estudo.

A coleta dos dados ocorreu em dois momentos. No primeiro momento, realizou-se a medida da estatura, massa corporal, das dobras cutâneas e pressão arterial sistêmica em repouso dos participantes. No segundo momento, reavaliou-se a pressão arterial sistêmica dos adolescentes que apresentaram valores aumentados na primeira avaliação.

Utilizou-se os procedimentos padronizados ${ }^{(10)}$ para definir a estatura e a massa corporal. Avaliou-se estatura pelo estadiômetro portátil marca WISO ${ }^{\circledR}$, com escala de $1 \mathrm{~mm}$ e a massa corporal com uma balança digital portátil marca $\mathrm{WISO}^{\circledR}$ com escala de medida de $100 \mathrm{~g}$. 
Usou-se um adipômetro científico $\left(\right.$ marca Cescorf $\left.{ }^{\circledR}\right)$ para realizar a medição das dobras cutâneas. Os pontos de reparo foram as dobras cutâneas subescapular, tricipital, suprailíaca, abdominal e panturrilha, seguindo padronização internacional para as medições ${ }^{(10)}$. Realizou-se três medidas não consecutivas das dobras cutâneas em cada ponto de reparo e calculou-se a média entre as medidas. Definiu-se o nível de adiposidade dos indivíduos a partir do somatório de dobras cutâneas nos cinco pontos de reparo.

Aferiu-se a pressão arterial sistêmica através do método auscultatório(12) utilizando esfigmomanômetro aneróide (marca BD). A aferição foi realizada no braço direito com os escolares sentados em repouso mínimo de 5 minutos. Utilizaram-se manguitos apropriados correspondentes a $40 \%$ da circunferência do braço e pelo menos $80 \%$ de seu comprimento $^{(12)}$. Para classificar os adolescentes, empregouse os valores propostos pelo National High Blood Pressure Education Program Working Group on High Blood Pressure in Children and Adolescents ${ }^{(12)}$ conforme o sexo, idade e percentil da estatura, sendo considerados os indivíduos pré-hipertensos com PAS e/ou PAD $\geq$ percentil $90^{\circ} \mathrm{e}<$ percentil $95^{\circ}$ e com valores de 120/80 $\mathrm{mmHg}$ e indivíduos hipertensos com a PAS e/ ou PAD $\geq$ percentil $95^{\circ}$. No caso dos adolescentes identificados com pré-hipertensão ou hipertensão no primeiro dia de coleta, uma nova avaliação da PAS e PAD foi realizada no dia posterior à primeira coleta. Nestes casos, os valores obtidos na segunda visita foram utilizados.

$\mathrm{Na}$ estatística descritiva, baseada em médias e desvios padrão, utilizou-se a descrição das variáveis contínuas e análise de frequência relativa para identificar as proporções de indivíduos com pré-hipertensão e hipertensão arterial. A comparação entre os sexos das proporções de indivíduos com valores normais e elevados de pressão arterial sistêmica foram obtidos através de testes de qui-quadrado.

A correlação parcial foi utilizada para analisar a associação entre o somatório de dobras cutâneas e a PAS e PAD. Nessas análises, estatura e idade foram inseridas como variáveis de controle. As análises foram realizadas por sexo, considerando um nível de significância de $\mathrm{p}<0,05$.

Este estudo foi aprovado pelo Comitê de Ética em Pesquisa da Universidade Federal do Paraná (Santa Catarina) com parecer $n^{\circ} 131.341$, seguindo as normas que regulamentam a pesquisa envolvendo seres humanos, do Conselho Nacional de Saúde (Resolução no 466/12). Participaram da pesquisa apenas os adolescentes cujos pais aceitaram e assinaram termo de consentimento livre e esclarecido (TCLE).

\section{RESULTADOS}

A amostra do presente estudo foi composta por 257 $(47,4 \%)$ adolescentes do sexo masculino e $286(52,6 \%)$ do sexo feminino, com média de idade de 14,76 $\pm 1,63$ anos. Caracterizou-se a amostra através das médias e desvio padrão da idade, estatura, massa corporal, dobras cutâneas do tríceps, subescapular, suprailíaca, abdominal, panturrilha, PAS e PAD para os adolescentes matriculados em escolas da rede pública de ensino da cidade de CuritibaPR (Tabela I).

Em relação às classificações da pressão arterial sistêmica, $83,2 \% \quad(n=452)$ dos adolescentes avaliados apresentaram valores normais, 9,2\% $\quad(n=50)$ foram considerados pré-hipertensos e $7,6 \%(n=41)$ considerados hipertensos.

Tabela I - Distribuição das variáveis analisadas segundo sexo dos adolescentes das escolas públicas. Curitiba-PR, 20102011.

\begin{tabular}{lccccc}
\hline \multirow{2}{*}{ Variáveis } & \multicolumn{2}{c}{ Masculino $(\mathbf{n = 2 5 7})$} & & \multicolumn{2}{c}{ Feminino $(\mathbf{n = 2 8 6})$} \\
\cline { 2 - 3 } \cline { 5 - 6 } & Média & DP $( \pm)$ & & Média & DP $( \pm)$ \\
\hline Idade (anos) & 14,71 & 1,61 & & 14,79 & 1,65 \\
Estatura (m) & 1,65 & 0,10 & & 1,59 & 0,06 \\
Massa corporal (kg) & 57,87 & 13,80 & & 53,49 & 10,15 \\
DC Tríceps (mm) & 12,09 & 5,58 & & 17,80 & 5,79 \\
DC Subescapular (mm) & 11,36 & 6,73 & & 15,04 & 6,77 \\
DC Suprailíaca (mm) & 15,92 & 11,50 & & 23,01 & 10,99 \\
DC Abdominal (mm) & 17,65 & 11,16 & & 25,26 & 10,25 \\
DC Panturrilha (mm) & 12,90 & 6,66 & & 18,88 & 6,62 \\
DC (mm) & 69,94 & 38,77 & & 100,01 & 36,69 \\
PAS (mmHg) & 111,65 & 9,66 & & 107,46 & 8,78 \\
PAD (mmHg) & 70,95 & 7,07 & & 70,84 & 6,68 \\
\hline
\end{tabular}

DP; desvio padrão; DC; dobra cutânea; PAS; pressão arterial sistólica; PAD; pressão arterial diastólica, $\sum$ DC; somatório de dobras cutâneas 


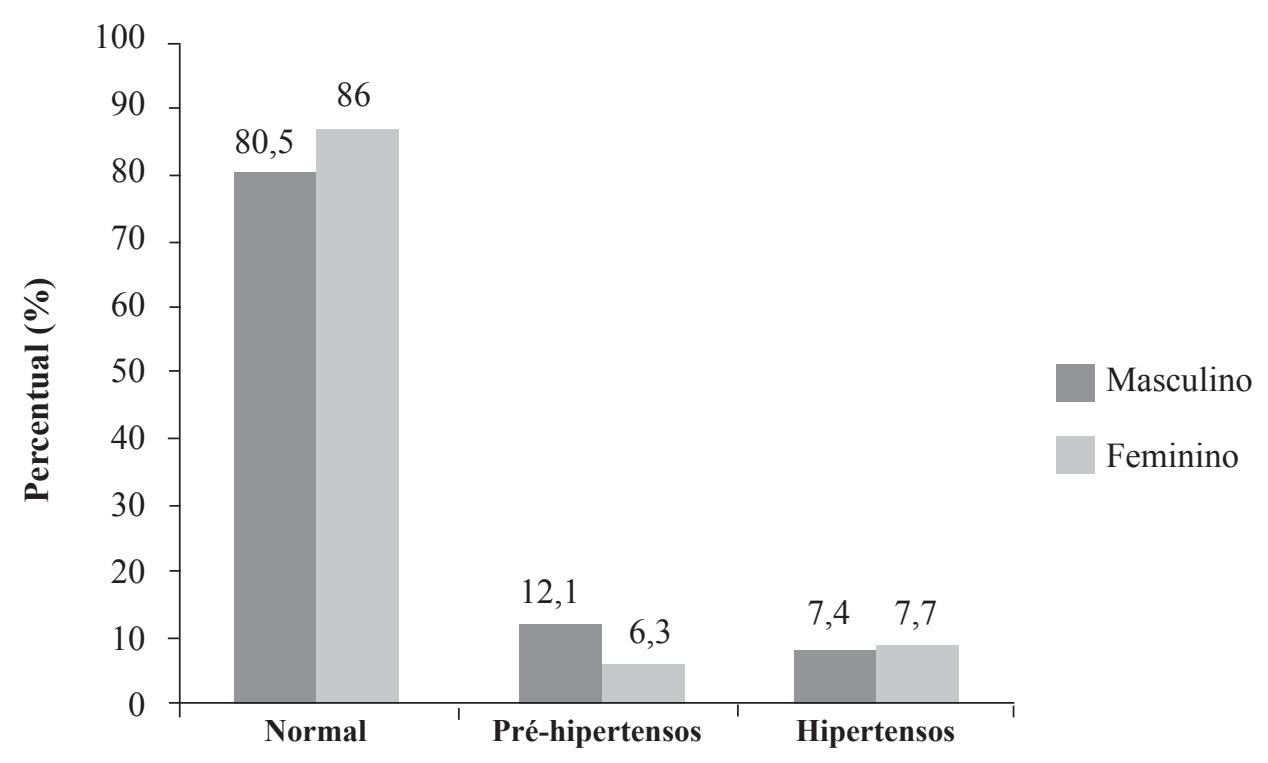

Figura 1 - Classificação dos valores de pressão arterial sistêmica segundo o sexo dos acadêmicos das escolas públicas. Curitiba-PR, 2010-2011.

A figura 1 demonstra a classificação da amostra segundo os valores de pressão arterial sistêmica em ambos os sexos. Não houve diferença significativa entre os sexos com os valores normais de pressão arterial sistêmica $\left(X^{2}=3,358 ; p=0,07\right)$, de pré-hipertensão $\left(X^{2}=3,449 ; p=0,06\right)$ e hipertensão $\left(X^{2}=0,639 ; p=0,22\right)$.

Em relação às análises de correlação parcial, foi observado que para o sexo masculino houve uma correlação significativa do somatório de dobras cutâneas com a PAS $(\mathrm{r}=0,18 ; \mathrm{p}<0,01)$ e com a PAD $(\mathrm{r}=0,14 ; \mathrm{p}<0,05)$. Para o feminino, também ocorreu correlação significativa com a PAS $(\mathrm{r}=0,15 ; \mathrm{p}<0,01)$ e com a PAD $(\mathrm{r}=0,19 ; \mathrm{p}<0,01)$. Os resultados demonstram que quanto maior o somatório de dobras cutâneas maior é a PAS e PAD, contudo, as correlações foram fracas.

\section{DISCUSSÃO}

Estudos realizados em várias regiões do país demonstraram prevalências superiores de adolescentes com hipertensão arterial sistêmica, com $11,8 \%{ }^{(7)}$ e $21,2 \%{ }^{(5)}$. Contudo, tais estudos realizaram a avaliação da pressão arterial sistêmica somente em um único dia, sendo que essa única avaliação pode superestimar as prevalências, uma vez que a pressão arterial sistêmica em altos níveis tende a diminuir nas próximas avaliações, devido a um efeito de acomodação gerado pela redução da ansiedade dos adolescentes referente à familiaridade com os procedimentos e o avaliador ${ }^{(12)}$.

Estudos que realizaram a medida da pressão arterial sistêmica em dois ou mais dias, assim como o presente estudo, demonstraram prevalências inferiores. Em consonância com esta afirmativa, estudos anteriores evidenciaram que 3,2\% de uma amostra de adolescentes apresentou hipertensão arterial sistêmica ${ }^{(13)}$ e que a prevalência desta pode variar de $2,8 \%$ para o sexo masculino e de $0,9 \%$ para o feminino ${ }^{(6)}$.

Sobre a correlação do somatório de dobras cutâneas com a pressão arterial sistêmica, outros estudos encontraram resultados semelhantes. Pesquisa realizada no Rio de Janeiro em 1998 com 646 adolescentes já alertava para uma relação entre o somatório de dobras cutâneas com a hipertensão arterial sistêmica, onde verificou uma correlação positiva do somatório de dobras cutâneas (tricipital e panturrilha) com a PAS $(\mathrm{r}=0,36)$ e com a PAD $(\mathrm{r}=0,30)$ no sexo masculino e com a PAS e PAD $(r=0,26)$ no sexo feminino ${ }^{(14)}$. Estudo transversal em Santa Cruz do Sul-RS encontrou, assim como na presente pesquisa, uma correlação fraca $(\mathrm{r}=0,30$ e $r=0,27)$ do somatório das dobras cutâneas tricipital e subescapular com PAS e PAD, respectivamente ${ }^{(15)}$. Esta correlação também foi encontrada em estudo com 1.253 adolescentes matriculados em escolas da rede pública e privada na cidade de Maceió-AL, detectaram correlação significativa entre a dobra cutânea do tríceps e a PAS $(r=0,25)$ e com a PAD $(r=0,24)$ em adolescentes de ambos os $\operatorname{sexos}^{(16)}$.

Tais estudos corroboram os achados da presente pesquisa, demonstrando que a elevação dos valores de PAS e PAD está associada com o aumento dos valores das dobras cutâneas. Entretanto, algumas diferenças metodológicas devem ser consideradas para explicar a variabilidade nos valores encontrados. 
No presente estudo, foram utilizados cinco pontos de reparo para o somatório de dobras, sendo que os demais estudos utilizaram somente uma ou duas dobras. Neste sentido, o protocolo de cinco dobras cutâneas parece representar melhor a quantidade total de gordura dos indivíduos por medir um maior número de segmentos corporais.

Ainda, nos demais estudos foram utilizadas análises de correlação simples, não havendo nenhum tipo de controle por outras variáveis que podem contribuir para o aumento da pressão arterial sistêmica. No presente estudo, houve controle pela idade, pois se sabe que a pressão arterial sistêmica tende a aumentar proporcionalmente com o passar dos anos(2). Ressalta-se então que a idade deveria ser considerada como variável de controle em estudos que demonstram prevalências e correlações da pressão arterial sistêmica $^{(17)}$.

Além disso, houve controle pela estatura dos participantes da pesquisa, uma vez que esta variável pode estar relacionada com o nível de maturação dos indivíduos. Assim, como a idade ${ }^{(17)}$, é usada nas tabelas normativas para classificação da pressão arterial sistêmica em indivíduos jovens $^{(12)}$.

Apesar disto, na presente pesquisa as correlações encontradas foram fracas. Isto se vincula ao fato de que apenas um indicador de risco pode representar uma limitação, uma vez que os valores aumentados de pressão arterial sistêmica são dependentes de uma combinação de fatores genéticos e ambientais. Estes fatores geralmente se apresentam de forma agregada, especialmente em famílias com estilo de vida pouco saudável ${ }^{(2,17)}$.

Contudo, foi demonstrado neste estudo um resultado consistente com a literatura, ratificando que elevados valores de pressão arterial sistêmica estão relacionados com maiores valores de dobras cutâneas. Esta identificação é importante, pois se sabe que indivíduos jovens com níveis de pressão arterial sistêmica mais elevada, mesmo que dentro de limites considerados normais, têm maiores chances de se tornarem adultos com valores aumentados de pressão arterial sistêmica ${ }^{(18)}$.

\section{CONCLUSÃO}

O somatório de dobras cutâneas apresentou relação direta com pressão arterial sistêmica nos adolescentes investigados.

\section{REFERÊNCIAS}

1. James PA, Oparil S, Carter BL, Cushman WC, Dennison-Himmelfarb C, Handler J, et al. Report from the Panel Members Appointed to the Eighth Joint
National Commitee (JNC 8). 2014 Evidence-Based Guideline for the Management of High Blood Pressure in Adults. JAMA. 2014;311(5):507-20.

2. Sociedade Brasileira de Cardiologia, Sociedade Brasileira de Hipertensão, Sociedade Brasileira de Nefrologia. VI Diretrizes Brasileiras de Hipertensão. Arq Bras Cardiol. 2010;95(Supl 1):1-51.

3. National High Blood Pressure Education Program Coordinating Committee. The Seventh Report of the Joint National Committee on Prevention, Detection, Evaluation, and Treatment of High Blood Pressure: the J.N.C. 7 report. JAMA. 2003;289(19):2560-72.

4. Cureau FV, Reichert FF. Indicadores antropométricos de obesidade como preditores de pressão arterial elevada em adolescentes. Rev Bras Cineantropom Desempenho Hum. 2013;15(3):338-49.

5. Araújo FL, Monteiro LZ, Pinheiro MHNP, Silva CAB. Prevalência de fatores de risco para a hipertensão arterial em escolares do município de Fortaleza, CE. Rev Bras Hipertens. 2010;17(4):203-9.

6. Beck CC, Lopes AS, Pitanga FJG. Indicadores antropométricos como preditores da pressão arterial elevada em adolescentes. Arq Bras Cardiol. 2011;96(2):126-33.

7. Christofaro DGD, Ritti-Dias RM, Fernandes RA, Polito MD, Andrade SM, Cardoso JR, et al. Detecção de hipertensão arterial em adolescentes através de marcadores de adiposidade geral e abdominal. Arq Bras Cardiol. 2011;96(6):465-70.

8. Monteiro CA, Mondini L, Souza ALM, Popkin BM. Da desnutrição para a obesidade: a transição nutricional no Brasil. In: Monteiro CA, editor. Velhos e novos males da saúde no Brasil. $2^{\mathrm{a}}$ ed. São Paulo: Hucitec; 2000. p. 247-55.

9. Flegal KM, Ogden CL, Yanovski JA, Freedman DS, Shepard JA, Graubard BI, et al. High adiposity and high body mass index-for-age in US children and adolescents overall and by race-ethnic group. Am J Clin Nutr. 2010;91(4):1020-6.

10. Guedes DP, Guedes JERP. Manual prático para avaliação em educação física. São Paulo: Manole; 2006.

11. Conde WL, Monteiro CA. Body mass index cut off points for evaluation of nutritional status in Brazilian children and adolescents. J Pediatr (Rio J). 2006;82(4):266-72.

12. National High Blood Pressure Education Program Working Group on High Blood Pressure in Children and Adolescents. Pediatrics. 2004;114(2):555-76. 
13. Rosa MLG, Mesquita ET, Rocha ERR, Fonseca VM. Índice de massa corporal e circunferência da cintura como marcadores de hipertensão arterial em adolescentes. Arq Bras Cardiol. 2007;88(5):573-8.

14. Costa RS. Relação entre sobrepeso, adiposidade e distribuição de gordura com a pressão arterial de adolescentes no município do Rio de Janeiro. Rev Bras Epidemiol. 1998;1(3):268-79.

15. Burgos MS, Reuter CP, Burgos LT, Pohl HH, Pauli LTS, Horta JA, et al. Uma Análise entre Índices Pressóricos, Obesidade e Capacidade Cardiorrespiratória em Escolares. Arq Bras Cardiol. 2010;94(6):788-93.

16. Souza MGB, Rivera IR, Silva MAM, Carvalho ACC. Relação da obesidade com a pressão arterial elevada em crianças e adolescentes. Arq Bras Cardiol. 2010;94(6):714-9.
17. Rosa AAA, Ribeiro JP. Hipertensão arterial na infância e na adolescência: fatores determinantes. J Pediatr (Rio J). 1999;75(2):75-82.

18. Din-Dzietham R, Liu Y, Bielo MV, Shamsa F. High blood pressure trends in children and adolescents in national surveys, 1963 to 2002. Circulation. 2007;116(13):1488-96.

\section{Endereço para correspondência:}

Rodrigo Bozza

Universidade Federal do Paraná - UFPR

Departamento de Educação Física - Centro de Pesquisa em Exercício e Esporte

Rua Coração de Maria, 92

Bairro: BR 116, km 95

CEP: 80215-370 - Curitiba - PR - Brasil.

E-mail: rdbozza@gmail.com 